\title{
Improvement of the procurement process using the integrated tender recommendation system
}

\author{
Zinaida K. Avdeeva \\ Senior Researcher, Trapeznikov Institute of Control Sciences; \\ Associate Professor, Department of Innovation and Business in Information Technologies \\ National Research University Higher School of Economics \\ Address: 65, Profsoyuznaya Street, Moscow, 117997, Russian Federation \\ E-mail: avdeeva@hse.ru
}

\section{Alexander A. Utrobin}

Bachelor in Business Informatics

National Research University Higher School of Economics Address: 20, Myasnitskaya Street, Moscow, 101000, Russian Federation

E-mail: alexanderutrobin11@gmail.com

\section{Ilya Y. Lykov}

MSc Program Student, Faculty of World Economy and International Affairs

National Research University Higher School of Economics

Address: 20, Myasnitskaya Street, Moscow, 101000, Russian Federation

E-mail: lykov.ilya@gmail.com

\section{Abstract}

Currently, tender procedures occupy an important place in the work of the procurement department of any company. Most of the modern recommendation services operate on web-platforms. Implementation of a tendering system in a company can increase the level of maturity of the procurement process and will not require serious changes in the structure of the processes. This article is devoted to the study of the structure of an integrated tender recommendation system. The integrated tender recommendation system is based on procedures prescribed by federal laws, aggregated tenders from different e-trade sites (presenting state and commercial platforms); it offers to its users additional services.

The main purpose of the study is to develop an effective model of an integrated tender recommendation system. In the description part of this article, we present information on peculiarities of the tender procedure in the Russian Federation and modern advisory services are considered. The functional advantages of an integrated system in comparison with the web platform are set out. The structure of the system is designed using several approaches. Using the IDEF0 methodology, a functional model of the system that reflects the work of processes has been developed and described. The operation of the main system and subsystems has been analyzed using the projected diagrams of the DFD methodology. A mathematical model of dynamic filtering of tenders for creating recommendations to users is described. Relying on the basic principles of collaborative filtering and, with the help of appropriate algorithms, the integrated system gives recommendations to users and determines the probability of success in a particular tender. Application of such technology of tenders is possible in companies of different scale. The developed structure of the integrated system and filtering methods for recommendations are based on the basic principles of a new international trend - e-tendering. 
Key words: recommendation services, tender, electronic trading platform, integrated system, dynamic filtration.

Citation: Avdeeva Z.K., Utrobin A.A., Lykov I.Y. (2017) Improvement of the procurement process using the integrated tender recommendation system. Business Informatics, no. 4 (42), pp. 29-39. DOI: 10.17323/1998-0663.2017.4.29.39.

\section{Introduction}

$\mathrm{W}$ ith the development of information technology, most of the world's countries have switched to electronic trading platforms. Tender is an integral part of the governmental and commercial sectors of the economy. There are many electronic trading platforms (ETP) and recommendation web services that collect requirements of different categories and deliver information through queries. Such sites and services also offer additional services.

The efficient operation of an enterprise's purchasing department is directly dependent on automation of the tender process. In "routine" tenders, employees regularly make mistakes during preparation of applications or when assembling a package of documents; they also may miss important changes in the specifications of terms or periods. In order to avoid errors and increase the purchasing department's efficiency, an integrated tendering system should be implemented at the enterprise. As a result, employees will be able to upload documents into the system and conduct all operations related with the tender, from planning to signing contracts with customers. An integrated system of tendering combines the functions of classical ETP and recommendation web services. It also allows users to automate "routine" tenders and proposes the recommended tenders. The system always provides warnings regarding changes in the terms of the application, the timing and composition of documents.

Modern ways of tender processing represent the object of this research, and the functioning of an integrated tender recommendation system is the subject of this study.

This article has the following structure. In the first section, peculiarities of competitions (tenders) in the Russian Federation are considered. In the second section, the work of modern advisory services is analyzed. In the third section, the model of an integrated tender recommendation system is presented, an effective structure of the system is designed, and the functions and subsystems are defined. This section also provides a dynamic filtration system, which is based on certain mathematical methods used in recommendation services. Finally, in the fourth section, the results of the study are presented.

\section{Features of electronic tenders in Russia}

A tender is a method of competing for a contract among which is compliant with state regulations and, under certain conditions, depends on the internal rules of the trading platform. As a result of tenders, the process of concluding a contract between the customer and the winner is performed. With this type of trading operation, the customer makes a choice from a set of proposals from different companies. As a result, the selected company undertakes an obligation to perform work, service or delivery of some goods to the customer. In Russian legal acts (http://zakupki-tendery.ru/zakonodatelstvo), the concept of "tender" is not officially used; instead we find the term "competition".

Modern web sites designed for holding trades are presented in three fields: state order, municipal order and commercial order. Each area is 
related with a certain organizer and the inherent specialized selection criteria. The organizers include government agencies, local governments and business entities. The special criteria are value for money, information about orders performed by the participant earlier, as well as additional conditions provided by the participants in the application. Within these three fields, various forms of the competition are available. The most frequently used tender forms are an open tender, a closed tender, a specialized closed auction, a request for quotations, a purchase from one supplier, and a request for proposals.

Since January 2011, all government customers are required to register and conduct tenders on one of five federal state electronic platforms: Sberbank AST, Rosetorg, OSET, ETP, MMBV "Goszakupki". Since January 2014, the Federal Law of 05.04.2013 No. 44-FZ "On the contract system in the sphere of procurement of goods, works and services to provide for state and municipal needs" is applied. Now, in addition to the tender procedures, analysis of previous trades is conducted in the electronic trading platforms, the statistics on the results of the competitions are posted, and information on planning and orders is provided. The federal law from 18.07.2011 No. 223-FZ "On procurement of goods, works and services by certain types of legal entities" regulates activities of legal entities - state owned corporations, monopolies, autonomous institutions, economic societies, companies of small and medium business. Before the appearance of the law, the activities of legal entities and the organization of competition were regulated through a variety of legal acts.

\section{Modern recommendation services for tender search and planning}

In order to find potential business partners, companies may use search systems, web aggregators of state trading platforms, industry related and universal sites, free and commercial bidding sites, forums, e-trading platforms of debtors' property sales. With the development of information technologies, most modern ETPs provide for their users certain additional functions of planning and holding tenders. However, at present only commercial systems present comprehensive services for tender search and planning. These services solve a number of problems, such as the huge number of online sites of competitions, specific interface of systems, weak return from search, complexity of monitoring the changes in tender conditions, as well as organizing documents.

Let us consider the results of the survey in large companies in Australia, the UK, China, the USA and Japan, represented in [1]. There are three architectural types of modern electronic trading platforms. The most popular is an architecture based on the principal. In this case, the state acts both as a customer and as an organizer of the system. The second type is an architecture based on an independent third party. A vivid example of such a system is a commercial electronic trading platform. The third type is an architecture based on responsibilities distributed between independent third parties. In such systems, functions are performed by different legal entities. For example, one company deals with the security of the system, another is responsible for the electronic digital signature. At present, the advisory systems for the search and planning of the competition are presented in the last two types of architecture.

\section{An integrated tender recommendation system}

Improving the functioning of the purchasing department of a company can be carried out with the help of an integrated tendering system. For modern Russia, we usually find that competitions related recommendation services provide their services through a web site and cloud computing. The integrated system has several advantages: minimization of the probability of system failures at critical times, con- 


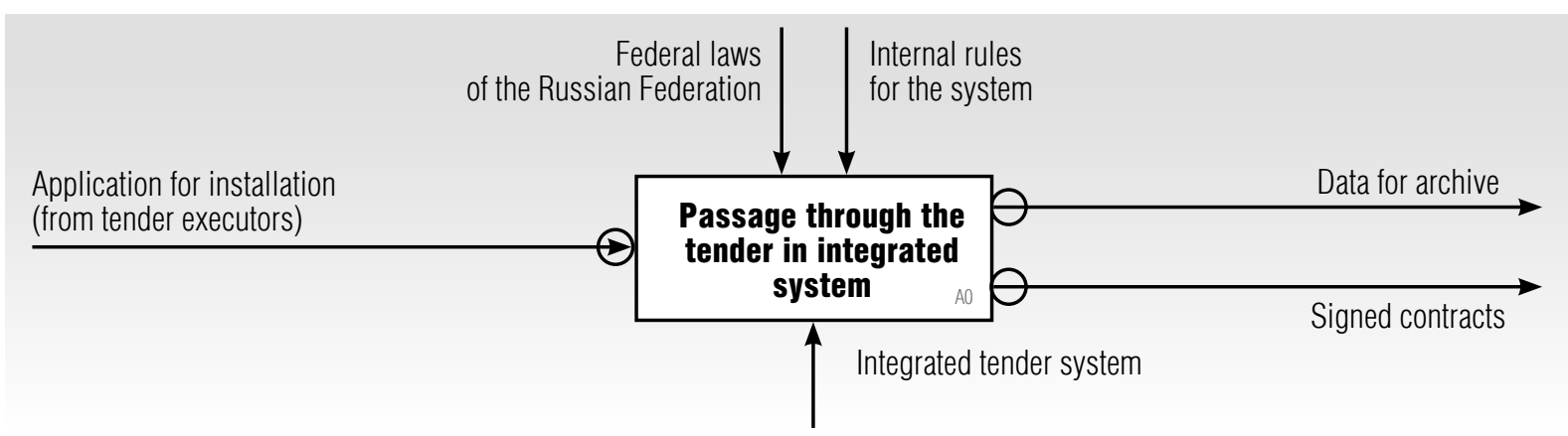

Fig. 1. Context diagram of the tender process in the integrated system

fidentiality of correspondence and control over access to tender information, minimization of financial and legal risks associated with third parties, using an effective system of tender filtering, application of an individual approach to each company registered in the system.

In general, the development of online bidding systems for large companies aims at improving the efficiency of the procurement process through open tenders. For medium-sized businesses, this means an opportunity to expand partnerships and find new business partners. For a small business, it is a convenient search system and an effective system of recommendations which the company can use to determine tenders where it is most likely to win, to view its statistics and to develop a specific strategy for activities of the procurement department.

\subsection{The process of passing through the tender in an integrated tender system}

The formalization of passing through the tender is constructed on the basis of the IDEF0 methodology. Figure 1 shows a contextual diagram of a tender passage process within an integrated system, as well as related additional information. Entrance objects are applications from the executors; the controls are the Federal Law of the Russian Federation and internal rules of the system; the mechanism is an integrated tender system; and the output objects are data for the archive and signed contracts.

The main functions provided by the integrated system are registration in the system, manual search, automatic search, rendering additional services, reporting the tender passage and archiving (Figure 2).

Let us consider the diagram by its stages. Registration in the system involves working with approved applications received from companies. This function is performed using an integrated system and is based on the company's internal rules. As a result, the function provides information for the file and user's ID. Companies registered in the system can perform a manual search through appropriate inquiries. Automatic search involves recommendations to users, providing information on tenders that are important for the company, as well as automatic submission of applications for regular competitions and setting up an electronic signature in the user's account. The function of providing additional services implies an individual approach to users, if necessary - assistance in drawing up an application for a tender, reminders related with changes in terms and conditions, as well as the preparation of a package of documents. The function of forming a package of documents uses the results of previous functions - the results of manual and automatic searches and additional services. The function of passing through the tender implies working with applications and packages of documents received from suppliers. With the help of the archiving function, data analysis is performed, which is necessary for the automatic search function. In addition, the data to be used for further work are archived. 


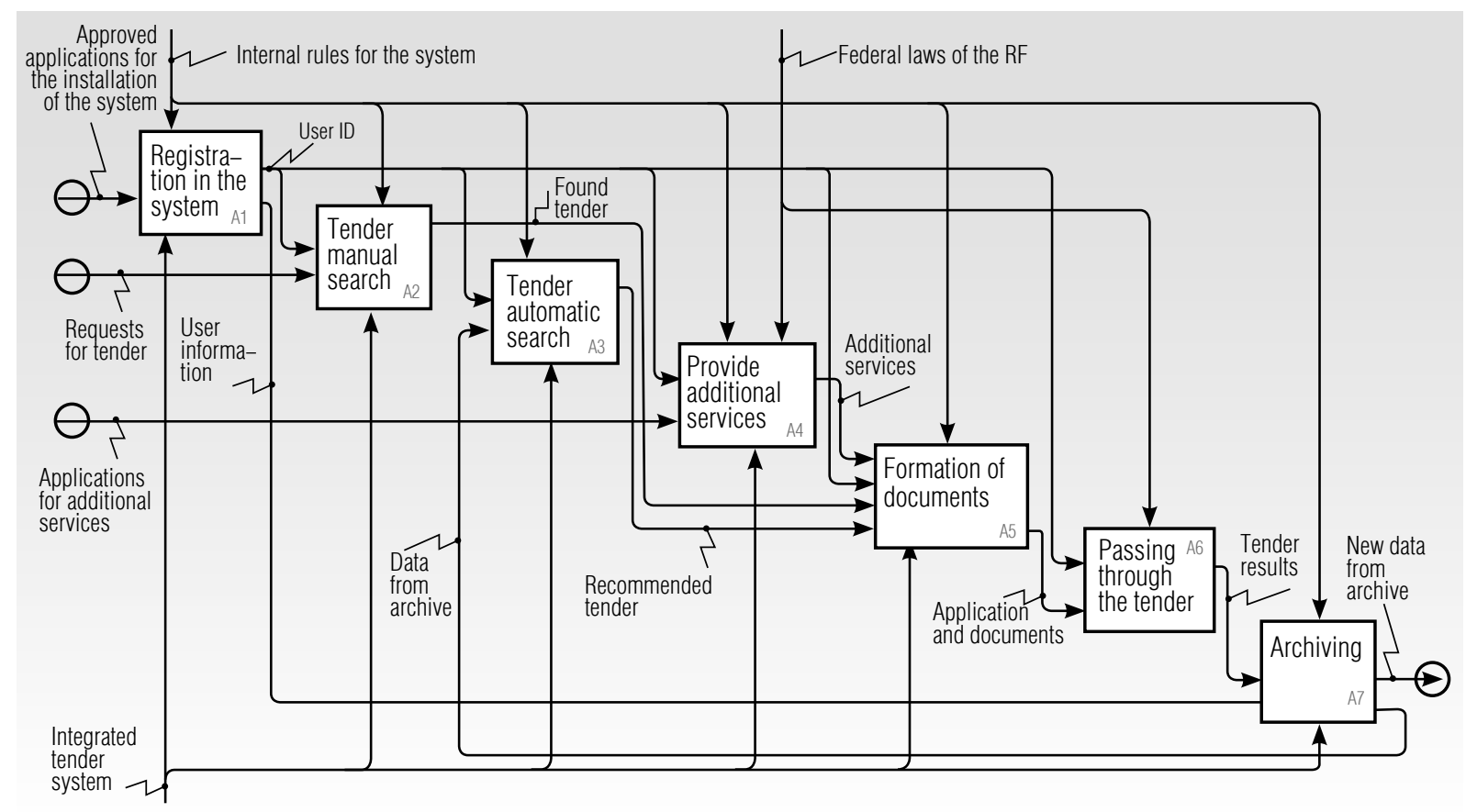

Fig. 2. The first level diagram of the tender process in an integrated system

\subsection{Analysis of data flows in an integrated system}

An integrated system implies working with executors of future and current competitions, as well as interaction with the management of the system. A relevant context DFD diagram is presented in Figure 3. From registered users, the system obtains information about the company, then on the basis of joint work in a personal account certain statistics supporting the recommendation system are generated. From the system, companies receive necessary information on tenders. From the management, the system receives internal rules, and management receives data for the archive.

The context diagram shows all the subsystems of the integrated tendering system (Figure 4). The archive subsystem performs storage of information on transactions and customers, as well as their search in the data warehouse. The search subsystem allows users to search and sort tenders. The search may be carried out using the manual method. It is also possible to select tenders according to required criteria among tenders recommended by the system. The subsystem of additional services provides the user with the opportunity to choose necessary services with the help of which the probability of winning the tender may be increased. The subsystem of the tender passage performs interaction of the installed program with state and commercial sites. Through a well-designed interface, users can go directly to the requirements of the integrated system. The dynamic filtering subsystem is looking for tenders recommended for each company on an individual basis, relying on mathematical methods and

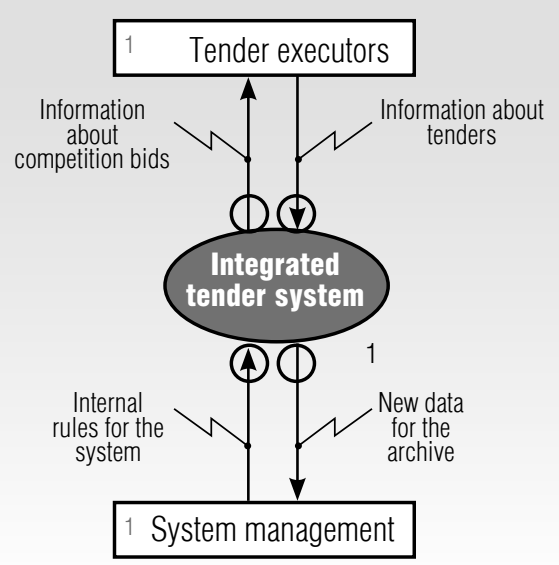

Fig. 3. Context diagram of the integrated tender system 


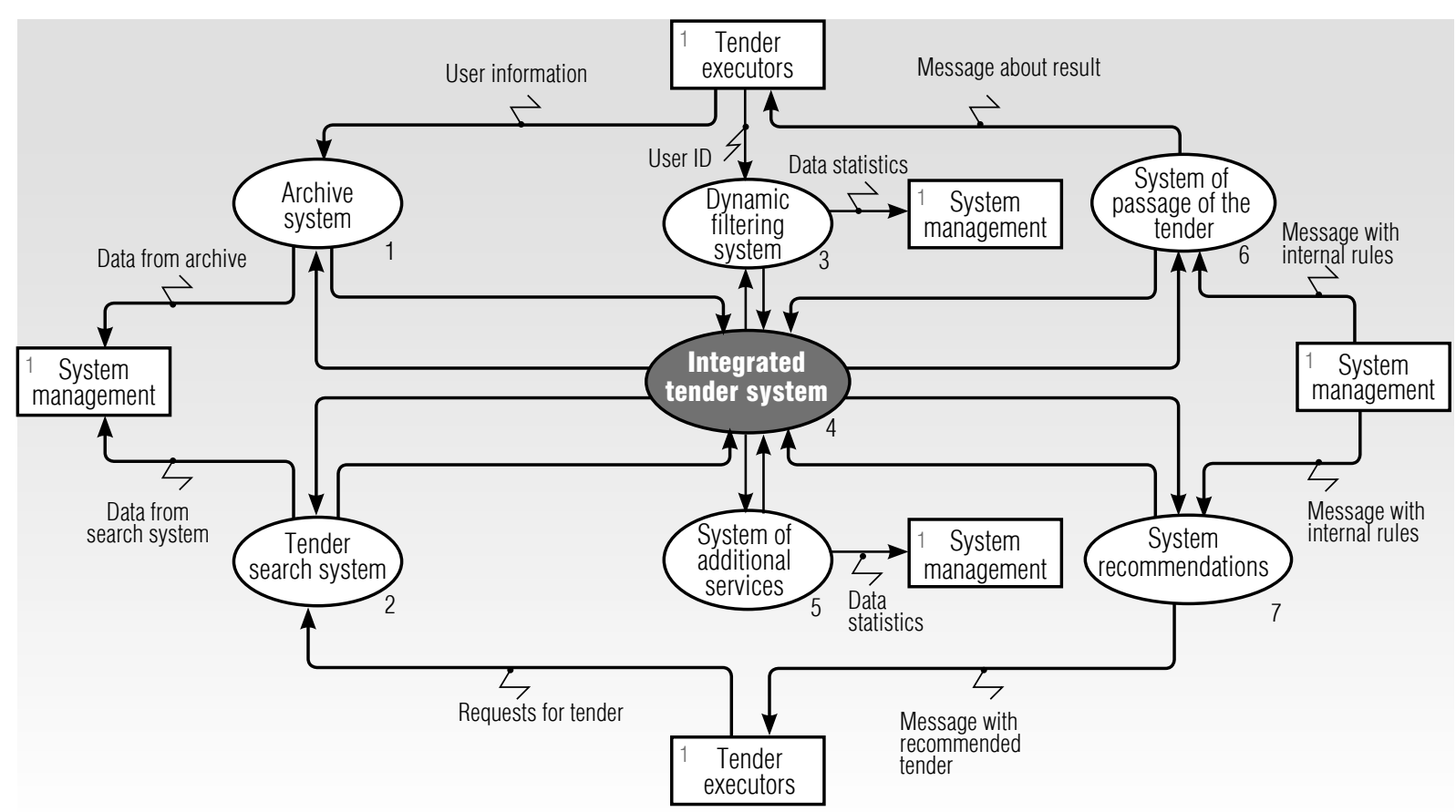

Fig. 4. Zero-level diagram of the integrated tender system

statistics generated in the archive system. The recommendation subsystem provides the user with the results of the work under a dynamic filtering system. If the user decides to conduct an automatic compilation and send regular requests for tenders, the recommendation system performs this work with the help of a pre-defined algorithm and digital signature.

\subsection{Dynamic filtering subsystem}

The maturity of the company's procurement process is determined by the ability to automate part of the processes, thus saving resources of the enterprise [2]. The electronic format makes it possible to avoid transaction costs arising during the search for a suitable supplier. In addition, the tender format opens up a number of possibilities for contracting companies in their search for customers. However, the process of searching for a customer can be quite timeconsuming and requires collection of large amounts of information. At this stage, the likelihood for both sides to make the wrong choice is increased. In this part of the work, a model that allows automatic selection of the tender is proposed. The model is based on the use of the basic principles of an advisory service. The key difference is that the method is applied for selection of a tender in which the contractor can participate. It is based on the principle of collaborative filtering: recommendations are based on the history of the estimates made both by a certain user and other users.

To start the recommendation system, users should assess the tenders in which they participated through this program. This condition is mandatory, as further recommendations will be based on these data. To evaluate different tenders, users should provide a certain set of characteristics. Ratings for each parameter are in the range of from 1 to 5 .

Among the characteristics examined are the following:

$\downarrow$ stability of documents status;

$\downarrow$ stability of application conditions;

$\downarrow$ stability of terms;

$\downarrow$ level of feedback with the customer; 
$\checkmark$ degree of the deal's transparency;

$\downarrow$ correctness of application conditions.

Relying on estimations for each of the listed characteristics, the overall assessment of the tender is formed, calculated by the weighted average formula:

$$
\bar{x}=\frac{\sum_{i=1}^{n} w_{i} x_{i}}{n},
$$

where $\bar{x}-$ score assigned by the user for a particular tender;

$x_{i}$ - score related with the $i$-th characteristic, $i=1, \ldots, n$;

$w_{i}$ - weight of the $i$-th characteristic, $i=1, \ldots$, $n$;

$n$ - number of characteristics.

The purpose of the algorithm is to generate a recommendation for each contractor. The recommendation is a tender offer which may be interesting for this executor. The system makes a recommendation, predicting the assessment that the user would put for a particular tender on the basis of its previous estimates. It is important that when logging into the system the user specifies the area of its activity for selection of the appropriate proposal.

The simplest metric that enables us to estimate proximity of two elements is the Euclidean distance between vectors. Obviously, that the smaller this value is, the closer the elements are and thus the more similarities they have. Relying on this certain recommendation, collection may be arranged. However, this method is not the only one. A better choice is possible using calculations that are more complex. Let us consider several possible algorithms for predicting the evaluation.

First, let us consider more advanced filtering methods. There is a set of executors $(u \in \mathrm{U})$ and a set of tenders suitable to the particular executor $(t \in \mathrm{T})$. On the basis of the processed information, the system makes a recommendation $e_{u t}$.

The first algorithm is associated with clustering of users. It is necessary to introduce the function of similarity $\operatorname{sim}(u, v)$, where $u$ is the selected user of the system, and $v$ is a user compared with the first one. This function shows how similar are scoring histories of the two users.

This function can be set in several ways. For example, it can be characterized as a percentage of tenders in which the two companies participate, or a share of tenders for which the companies have matched estimates. Further, users are divided into clusters, relying on the "like with like" principle: $u \rightarrow \mathrm{G}(u)$.

The predicted user's score of a certain tender is calculated as the average of all of the cluster to which the user belongs:

$$
\widehat{e_{u t}}=\frac{1}{|\mathrm{G}(u)|} \sum_{v \in \mathrm{G}(u)} e_{v t},
$$

where $\widehat{e_{u t}}-$ predicted score of tender $t$ by user $u, t \in \mathrm{T}, u \in \mathrm{G}(u)$;

$|\mathrm{G}(u)|-$ the number of users in a cluster, for which user $u$ belongs;

$e_{v t}$ - score of tender $t$ by user $v$ (another user of the cluster), $t \in \mathrm{T}, v \in \mathrm{G}(u)$;

$\mathrm{T}-\mathrm{a}$ set of tenders;

$\mathrm{G}(u)$ - a set of users of the cluster.

Let us consider another "user-based" algorithm which can be used to create recommendations [4]. We give a formula for predicting the evaluation. Here we also use the similarity function that was mentioned earlier:

$$
\widehat{e_{u t}}=\overline{e_{u}}+\frac{\sum_{v \in \mathrm{U}} \operatorname{sim}(u, v)\left(e_{v t}-\overline{e_{v}}\right)}{\sum_{v \in \mathrm{U}} \operatorname{sim}(u, v)},
$$

where $\widehat{e_{u t}}-$ predicted score of tender $t$ by user $u, t \in \mathrm{T}, u \in \mathrm{G}(u)$;

$\overline{e_{u}}$ - average typical score of user $u, u \in \mathrm{G}(u)$;

$\overline{e_{v}}-$ average score of a similar user $v, v \in \mathrm{G}(u)$;

$e_{v t}-$ actual score of tender $t$ by a similar user $v$, $t \in \mathrm{T}, v \in \mathrm{G}(u)$;

$\operatorname{sim}(u, v)$ - function of similarity of scores of the users $u$ and $v, u \in \mathrm{G}(u), v \in \mathrm{G}(u)$;

$\mathrm{T}-\mathrm{a}$ set of tenders;

$\mathrm{G}(u)$ - a set of users of the cluster.

Another possible algorithm of estimation is based on the principle of singular decomposition [4]: 


$$
\begin{gathered}
\widehat{e}_{u t}(\beta)=p_{u}^{\mathrm{T}} q_{t}, \\
\beta=\left\{p_{u} ; q_{t} \mid u \in \mathrm{U}, t \in \mathrm{T}\right\},
\end{gathered}
$$

where $\widehat{e_{u t}}(\beta)-$ predicted score of tender $t$ by user $u, t \in \mathrm{T}, u \in \mathrm{G}(u)$;

$p_{u}$ - possible score of user $u, u \in \mathrm{U}$;

$q_{t}$ - possible score of tender $t, t \in \mathrm{T}$;

$\beta-$ a couple of scores (possible score of user $p_{u}$ and tender $q_{t}$ );

$\mathrm{T}$ - a set of tenders;

$\mathrm{U}-\mathrm{a}$ set of users.

In this case, $p_{u}$ and $q_{t}$ are parameters that should be evaluated. It is necessary to optimize the quality of future predictions:

$$
\left(\widehat{e_{u t}}(\beta)-e_{u t}\right)^{2} \rightarrow \min ,
$$

where $\widehat{e_{u t}}(\beta)-$ predicted score of tender $t$ by user $u, t \in \mathrm{T}, u \in \mathrm{G}(u)$;

$e_{u t}-$ actual score of tender $t$ by user $u, t \in \mathrm{T}$, $u \in \mathrm{G}(u)$;

$\beta-$ a set of possible scores of user $u$ and ten$\operatorname{der} t$

$\mathrm{T}-$ a set of tenders;

$\mathrm{U}-\mathrm{a}$ set of users.

While the system has a number of previous estimates, we can speak about availability of a training sample. Let us rewrite (1), performing regularization:

$$
\sum_{u, t}\left(\widehat{e_{u t}}(\beta)-e_{u t}\right)^{2}+\lambda \sum_{\varphi \in \beta} \varphi^{2} \rightarrow \min ,
$$

where $\widehat{e_{u t}}(\beta)-$ predicted score of tender $t$ by user $u, t \in \mathrm{T}, u \in \mathrm{G}(u)$;

$e_{u t}-$ actual score of tender $t$ by user $u, t \in \mathrm{T}$, $u \in \mathrm{G}(u)$;

$\lambda$ - regularization parameter, $\lambda>0$;

$\varphi-$ stabilizing functional;

$\beta-$ a set of possible scores of user $u$ and ten$\operatorname{der} t$

$\mathrm{T}-$ a set of tenders;

$\mathrm{U}-\mathrm{a}$ set of users.

The feature of the regularization parameter $\lambda$ is that when $\lambda \rightarrow 0$ and the availability of errors in source data the solution of a correct task strives for the true solution of an appropriate incorrect task. The aim of the stabilizing functional $\varphi$ is to provide stability of solution of the task if source data are inaccurate.

Let us rewrite (2) for the purposes of the task under consideration:

$\sum_{u, t}\left(p_{u}^{\mathrm{T}} q_{t}-e_{u t}\right)^{2}+\lambda\left(\sum_{u}\left\|p_{u}\right\|^{2}+\sum_{t}\left\|q_{t}\right\|^{2}\right) \rightarrow \min$,

where $\beta-\mathrm{a}$ set of possible scores of user $\mathrm{u}$ and tender $t$;

$p_{u}$ - possible score of user $u, u \in \mathrm{U}$;

$\left\|p_{u}\right\|-$ norm of scores of user $u, u \in \mathrm{U}$;

$q_{t}-$ possible score of tender $t, t \in \mathrm{T}$;

$\left\|p_{u}\right\|-$ norm of scores of tender $t, t \in \mathrm{T}$;

$e_{u t}-$ actual score of tender $t$ by user $u, t \in \mathrm{T}$, $u \in \mathrm{G}(u)$;

$\lambda$ - regularization parameter, $\lambda>0$;

$\mathrm{T}$ - a set of tenders;

$\mathrm{U}-$ a set of users.

Thus, the problem has been reduced to finding the minimum of the functional. This allows us to find the most correct assessments for the predictions.

A separate advantage of the system so developed is not only automatic selection of the tender for the contractor, but also the evaluation of the probability of winning it. To calculate the probability, the system analyzes several criteria, assigning them ratings from 1 to 10 . Among the criteria are the following:

$\downarrow$ the degree of occupancy of the tender's executor;

$\downarrow$ user's experience in working with the system;

$\downarrow$ compliance with the business area of the tender;

$\downarrow$ production costs;

$\checkmark$ geographic distance (if the user is irrelevant for a particular situation, the system uses the first four criteria for assessing the probability of winning).

The probability is calculated as follows:

$$
\mathrm{P}(\mathrm{T})=\frac{\sum_{i=1}^{n} A_{i}}{\sum_{i=1}^{n} B_{i}} \cdot \frac{1}{n},
$$


where $\mathrm{P}(\mathrm{T})$ - probability of winning the tender; $A_{i}$ - the score assigned by the system to the tender, relying on the evaluation presented above;

$B_{i}$ - the maximal score;

$\sum_{i=1}^{n} A_{i}-$ the sum of the scores assigned to the tender relying on the criteria;

$\sum_{i=1}^{n} B_{i}$ - the maximal possible sum of the scores;

$n-$ the number of participants in the tender.

In addition to calculating the percentage of probability, the partitioning of the results by categories is provided (Table 1 ).

\section{Categories of probability of winning the tender}

\begin{tabular}{l|c|c}
\hline Categories & $\begin{array}{c}\text { Name } \\
\text { of the category }\end{array}$ & $\begin{array}{c}\text { Probability of } \\
\text { winning (\%) }\end{array}$ \\
\hline Category 1 & $\begin{array}{c}\text { The highest probability } \\
\text { of winning }\end{array}$ & $90-100$ \\
\hline Category 2 & $\begin{array}{c}\text { High probability } \\
\text { of winning }\end{array}$ & $70-89$ \\
\hline Category 3 & $\begin{array}{c}\text { Average probability } \\
\text { of winning }\end{array}$ & $50-69$ \\
\hline Category 4 & $\begin{array}{c}\text { Low probability } \\
\text { of winning }\end{array}$ & $30-49$ \\
\hline Category 5 & $\begin{array}{c}\text { The lowest probability } \\
\text { of winning }\end{array}$ & $0-29$ \\
\hline
\end{tabular}

Thus, in this part of the paper the following filtering techniques are discussed: estimation of important parameters of the tender and calculating a recommendation by the weighted average; simple metrics through Euclidean distance; the algorithm associated with clustering of users; a "user-based" algorithm; an algorithm based on the singular decomposition of the matrix. The probability of winning the tender is evaluated using the criteria and calculation of estimates using formula (3).

\section{The results of the study}

As a result of the study, an integrated recommendation tender system has been designed. Due to aggregation and data selection from dif- ferent ETPs, so-called "false" tenders can be identified and excluded from the set of tenders under consideration (lots with single provider, lots with special constraints, etc.). With the help of automated functions and recommendations, the level of transaction costs related with a tender executor is decreased. A high-quality integrated system includes the ability to store and process huge data sets.

This system can be used by companies of all sizes and all kinds of production activities. With the automation of processes in the purchasing department related to the tender, companies can save money. The effectiveness of the system depends on the elaborated structure of sub-systems, environs of the basic system functions, and on basic algorithms of the filtering system. The development of an integrated system was based on the basic principles of modern trends of e-tendering. The main features of this area are identified in [5]; they include minimization of legal and financial risks, minimization of errors in preparation of applications, and an effective tenders search system.

In the near future, e-tendering systems will be able to reach a higher level of quality. Many foreign authors speak about possible changes in the structure of the tender (for example, in [6]), and also examine the details of such changes. With the development of recommendation service technologies, new integrated systems will appear that are able to automate part of the process in the purchasing department, resulting in reduced costs of making a tender and procurement through tender. They will increase the level of maturity of the procurement process, will increase deals flows, will expand partner bases and will automate "routine" tenders.

\subsection{An example of the filtering system in operation}

Let us present some calculations illustrating the operation of the filtering system. For example, we consider an algorithm based on the "user-based" method. Suppose that there are 
two users $A$ and $B$. There is information on how they evaluated tenders in which they took part (Tables 2 and 3). It is necessary to predict what score will be assigned by user $A$ to the tender 6 .

Table 2.

\section{Evaluations of user A}

\begin{tabular}{c|c}
\hline Tender & Score \\
\hline Tender 1 & 5 \\
\hline Tender 2 & 3 \\
\hline Tender 3 & 4 \\
\hline Tender 4 & 2 \\
\hline Tender 5 & 5 \\
\hline
\end{tabular}

Evaluations of user B

\begin{tabular}{c|c}
\hline Tender & Score \\
\hline Tender 1 & 5 \\
\hline Tender 2 & 3 \\
\hline Tender 4 & 4 \\
\hline Tender 5 & 5 \\
\hline Tender 6 & 1 \\
\hline Tender 8 & 2 \\
\hline
\end{tabular}

Let us introduce a similarity function as a share of tenders for which the performers have matched estimates. Both customers participated in tenders 1, 2, 4 and 5. However, the evaluations coincided only for tenders 1,2 and 5. Thus the share of tenders with coinciding estimates for user $A$ is $3 / 5$.

Then we apply the formula for evaluation prediction and calculate an evaluation prediction of tender 6 by user A:

$$
\widehat{e_{u t}}=\overline{e_{u}}+\frac{\sum_{v \in \mathrm{U}} \operatorname{sim}(u, v)\left(e_{v t}-\overline{e_{v}}\right)}{\sum_{v \in \mathrm{U}} \operatorname{sim}(u, v)},
$$

where $\widehat{e_{u t}}-$ predicted score of tender $t$ by user $u, t \in \mathrm{T}, u \in \mathrm{G}(u)$;

$\overline{e_{u}}$ - average typical score of user $u, u \in \mathrm{G}(u)$;

$\frac{u}{e_{v}}-$ average score of a similar user $v, v \in \mathrm{G}(u)$; $e_{v t}-$ actual score of tender $t$ by a similar user $v, t \in \mathrm{T}, v \in \mathrm{G}(u)$;

$\operatorname{sim}(u, v)-$ function of similarity of scores of the users $u$ and $v, u \in \mathrm{G}(u), v \in \mathrm{G}(u)$;

$\mathrm{T}-\mathrm{a}$ set of tenders;

$\mathrm{G}(u)$ - a set of users of the cluster.

The average estimate of a typical user $\left(\overline{e_{u}}\right)$ we take as the average number of scores set out by the system. If we are afraid to overestimate the assessment, the geometric mean can be taken, since it is always less than the arithmetic mean, except when all the estimates are equal. In this case, it is 3.8, and in the case of using the geometric average it is 3.59 . For user $B$, the average $\left(\overline{e_{v}}\right)$ is 3.3. Making calculations, we get 3.83. This means that it is likely that user $A$ will assign the score 4 to tender 6 .

\subsection{An example of calculations}

To estimate the probability of winning the tender, the system uses a proposed technique assigning to each of the parameters a score from 1 to 10 . It is worth paying attention to the fact that different parameters are taken into account by a system in a different way. Once again we turn to the characteristics used in the calculation of probabilities and give some comments on the methodology for assigning the scores:

$\downarrow$ degree of tender executor occupancy (in this case, the relationship is reversed: the more loaded the executor is, the fewer points he receives for this feature);

user's experience in working with the system (direct relationship: the more experienced a user is, the higher is the score assigned to him);

$\downarrow$ correspondence of business activity to the subject of the considered tender (direct relationship);

$\checkmark$ cost of production (in this case, the relationship is reversed: the lower the cost of production from this manufacturer, the greater score it gets. The lower the production cost, the lower price the user may ask, and therefore, it has a better chance of getting the order, which should be reflected in the formula); 
$\checkmark$ geographical distance (if this parameter is irrelevant for a particular situation, the system uses the first four criteria for assessing the probability of winning. There is a direct relationship: the closer the performer is to the customer, the greater the probability of winning).

Assume that there are two firms participating in the tender $-A$ and $B$. Table 4 presents evaluations estimated by the system.

Scores assigned by the system

\begin{tabular}{c|c|c}
\hline Parameter & Firm A & Firm B \\
\hline Occupancy degree & 8 & 2 \\
\hline Experience & 7 & 4 \\
\hline Compliance & 10 & 7 \\
\hline Cost and price & 8 & 8 \\
\hline Geographical distance & 10 & 5 \\
\hline
\end{tabular}

Using formula (3) to define winning probability, we find that the wining probability of firm $\mathrm{A}$ is $43 \%$, and the wining probability of firm $\mathrm{B}$ is $26 \%$. It is obvious that company A has a greater chance to win, and with the growth of the number of participants the probability of winning will decrease.

\section{Conclusion}

Thus, in this article the efficiency of the integrated tender system has been considered. The current state of the federal legislation of the Russian Federation is analyzed, and specific features of conducting competitions are highlighted. The problems that may be solved using the system for companies of different scale are formulated. The structure of a recommendation service using IDEF0 and DFD methodologies has been designed. The mathematical methods and algorithms that may be applicable within the dynamic tenders filtering subsystem are considered.

\section{References}

1. Du R., Foo E., Nieto J.G., Boyd C. (2005) Designing secure e-tendering systems. Trust, privacy and security in digital business / S. Katsikas, J. López, G. Pernul (eds). Lecture Notes in Computer Science, vol. 3592. Springer, Berlin, Heidelberg, pp. 70-79.

2. KPMG (2011) Model'zrelosti zakupok. Analiz funktsii zakupok v rossiyskikh kompaniyakh [Procurement maturity model. Analysis of the procurement function in Russian companies]. Available at: https:// home.kpmg.com/ru/ru/home/insights/2011/06/model-of-procurement-maturity.html (accessed 01 September 2017) (in Russian).

3. Sarwar B.M., Karypis G., Konstan J.A., Reidl J. (2001) Item-based collaborative filtering recommendation algorithms. Proceedings of the 10th International World Wide Web Conference. Hong Kong, 1-5 May 2001. P. 285-295.

4. Jones T.M. (2014) Rekomendatel'nye sistemy. Chast' 1. Vvedenie v podkhody i algoritmy [Recommendation systems. Part 1. Introduction to approaches and algorithms]. Available at: https:// www.ibm.com/developerworks/ru/library/os-recommender1/ (accessed 15 March 2017) (in Russian).

5. Dawson E.P., Duncan W.D., Christensen S.A., Black P.J., Foo E., Du R., Boyd C.A., Nieto J. (2005) E-tendering - Security and legal issues: Research report. Brisbane: CRC Construction Innovation.

6. Lavelle D., Bardon A. (2009) E-tendering in construction: time for a change? Northumbria Working Paper Series: Interdisciplinary Studies in the Built and Virtual Environment, no. 2 (2), pp. 104-112. 